\title{
Validation of the Heart-Rate Signal Provided by the Zephyr BioHarness 3.0
}

\author{
Daniele Nepi, Agnese Sbrollini, Angela Agostinelli, Elvira Maranesi, Micaela Morettini, \\ Francesco Di Nardo, Sandro Fioretti, Paola Pierleoni, Luca Pernini, Simone Valenti, Laura Burattini \\ Department of Information Engineering, Università Politecnica delle Marche, Ancona, Italy
}

\begin{abstract}
The Zephyr BioHarness 3.0 (BH3) is a popular wearable system specifically designed for training optimization of professional athletes. BH3 provides the electrocardiogram (ECG_BH3) and the heart-rate signal (HRS_BH3). Aim of this study is to evaluate the reliability of HRS_BH3 to assess its clinical applicability to the general population for cardiac-risk evaluations. Data were acquired from 10 healthy subjects (age: $34 \pm 17$ years) during a 5-minutes rest. Since the tachogram represents the standard signal for studying the heart rate (HR) and its variability, ECG_BH3 was elaborated in order to get the tachogram (HRS_TG). HRS_BH3 and HRS_TG were compared in terms of mean HR (MHR, bpm), HR standard deviation (HRSD, bpm) and HRSD error (bpm). HRS_BH3 and HRS_TG provided comparable MHR (73.07 $\pm 15.53 \mathrm{bpm}$ vs $72.86 \pm 15.57$ bpm, respectively) while HRSD by HRS_BH3 was significantly lower than HRSD by HRS_TG $(4.51 \pm 2.29$ bpm vs $5.63 \pm 2.99$ bpm, respectively; $P=0.0043)$. HRSD error was significantly greater than zero $(0.20-3.00 \mathrm{bpm}$; $P=0.0043)$; moreover, it was strongly correlated to HRSD by HRS_TG $(\rho=0.82, P=0.0036)$. Thus, HRS_BH3 is appropriate only for sport applications based on MHR estimations, but not to clinical evaluations based on HRV measurements.
\end{abstract}

\section{Introduction}

Sudden cardiac death (SCD), defined as a "death occurring within 1 hour from the onset of symptoms in someone without a previously recognized cardiovascular abnormality" [1,2], remains one of the leading causes of death in developed countries [3]. Many cardiac defects, which may cause SCD, can be noninvasively identified by analyzing the electrocardiogram (ECG), which is the recording of the electrical activity of the heart, and the heart-rate (HR) signal (HRS), which is the sequence of the reciprocal of the cardiac-period represented as function of time. Indeed, abnormalities in cardiac repolarization, identifiable by analyzing the electrocardiographic ST segment and T wave [4-6], are also known to be linked to an increased risk of developing malignant ventricular arrhythmias that may degenerate in SCD [7-9]; and mean HR (MHR) and HR variability (HRV) parameters, obtained by analyzing the HRS, are universally recognized as indicators of SCD $[10,11]$. Monitoring of repolarization and HR indexes is usually performed by medical evaluation of standard ECG tracings or Holter ECG recordings, which require the subject to visit hospitals or doctor offices [12-14]. In case of transient abnormal cardiac episodes, such recordings may likely not include them.

Recently, wearable physical activity monitoring devices (such as heart-rate monitoring system, accelerometers, pedometers, and multiple-sensor devices) have become very popular among athletes for training optimization. In particular, cardiac monitoring devices are typically used for training optimization in terms of intensity, volume, duration and frequency. They usually measure instantaneous HR and sometimes record HRS. Occasionally, the most sophisticate devices also record and memorize ECG. Thus, the acquired data are the same that may be used for cardiac risk assessment. Consequently, if clinically reliable HRS and ECG are provided, such sensors could be used to gain important for cardiac risk evaluation not only in athletes [15-18] but in the general population, who could comfortably wears these devises at home, during daily activities or when feeling sick. The BioHarness 3.0 (BH3) by Zephyr is a popular wearable system specifically designed for training optimization of professional athletes. BH3 acquires several biological data among which the ECG and HRS. Thus, aim of the present study (which is part of larger project on BH3 validation) is to evaluate the reliability of HRS_BH3 in order to gain information on 
the possibility of using BH3 in the general population for contributing to the assessment of the risk of SCD.

\section{Methods}

\subsection{Zephyr BioHarness 3.0}

BH3 is a physiological monitoring telemetry device developed by Zephyr (www.zephyranywhere.com), now part of Medtronic. BH3 consists of a chest strap and an electronics module that attaches to the strap. The device may transmit by radio-frequency (transmission mode) or stores (logging mode) the data. While the first allows the live viewing of data; the second modality allows the later download of data. Among the output biological signals, there are ECG and HRS. Particularly, the single-lead ECG tracing (ECG_BH3; mV) is sampled at $250 \mathrm{~Hz}$, and HRS signal (HRS_BH3; bpm) is sampled at $1 \mathrm{~Hz}$.

\subsection{Data acquisition}

Clinical data were acquired from 10 healthy subjects (age: $34 \pm 17$ years) in order to have some levels of HRV. After wearing $\mathrm{BH} 3$ and set the device in logging mode, each subject was asked to stay still to acquire the signals in resting conditions. Eventually, ECG_BH3 and HRS_BH3 were downloaded on a PC for subsequent processing.

\subsection{Signal processing}

The tachogram is a signal defined as the RR-interval sequence expressed in function of consecutive number of beats (or time by multiplying by the mean RR interval). Since the tachogram represents the standard signal for studying HR and HRV, ECG_BH3 was elaborated in order to get the tachogram (HRS_TG), which is eventually compared to HRS_BH3. Particularly, ECG_BH3 was processed to detect the R-peak sequence using the Pan-Tompkins algorithm [19]. By considering the relation that exists between instantaneous HR and RR interval, the following equation

$$
H R=\frac{60}{\text { RR interval }}
$$

is used to compute the HR sequence from the $\mathrm{RR}$ sequence.
All processing procedures were implemented in MATLAB.

\subsection{Signal comparison}

Comparison between HRS_BH3 and HRS_TG was accomplished in terms of mean HR (MHR, bpm), HR standard deviation (HRSD, bpm) and HRSD error (bpm), here defined by the following equation:

$$
H R S D=H R S D_{B H 3}-H R S D_{T G}
$$

where $\mathrm{HRSD}_{\mathrm{BH} 3}$ and $\mathrm{HRSD}_{\mathrm{TG}}$ represent $\mathrm{HRSD}$ values provided by HRS_BH3 and HRS_TG, respectively.

MHR and HRSD values provided by HRS_BH3 and HRS_TG were compared using the t-test. Association between HRSD error and HRSD by HRS_TG were evaluated by computing of the Pearson's correlation coefficient $(\rho)$. Statistical significance level was set at 0.05 for all tests.

\section{Results}

An example of qualitative results is reported in Fig.1 that shows HRS_BH3 and HRS_TG signals of subject $n$. 2. Although HRS_TG and HRS_BH3 follow the same trend, HRS_TG is characterized by higher and more frequent amplitude variations (higher high-frequency content).

The quantitative results relative to each individual are reported in Table 1. Globally, HRS_BH3 and HRS_TG provided comparable MHR (73.07 \pm 15.53 bpm vs 72.86 \pm 15.57 bpm, respectively; $\mathrm{P}=0.1299$ ) while HRSD by HRS_BH3 was significantly lower than HRSD by HRS_TG (4.51 \pm 2.29 bpm vs 5.63 \pm 2.99 bpm, respectively; $\mathrm{P}=0.0043)$. HRSD error was always positive (from $0.20 \mathrm{bpm}$ to $3.00 \mathrm{bpm}$, Table 1), and thus significantly greater than zero $(\mathrm{P}=0.0043)$. Moreover, HRSD error was strongly correlated to HRSD by HRS_TG $(\rho=0.82, P=0.0036)$, as shown in Fig.2.

\section{Discussion}

The work presented in this paper, which represents the first part of larger project on $\mathrm{BH} 3$ validation, is finalized to evaluate reliability of cardiac signals provided by $\mathrm{BH} 3$ in order to gain information on the possibility of using $\mathrm{BH} 3$ in the general population for contributing to the assessment of the risk of SCD. To this aim, ECG_BH3 was elaborated in order to get HRS_TG, which is eventually compared to HRS_BH3, this latter signal 


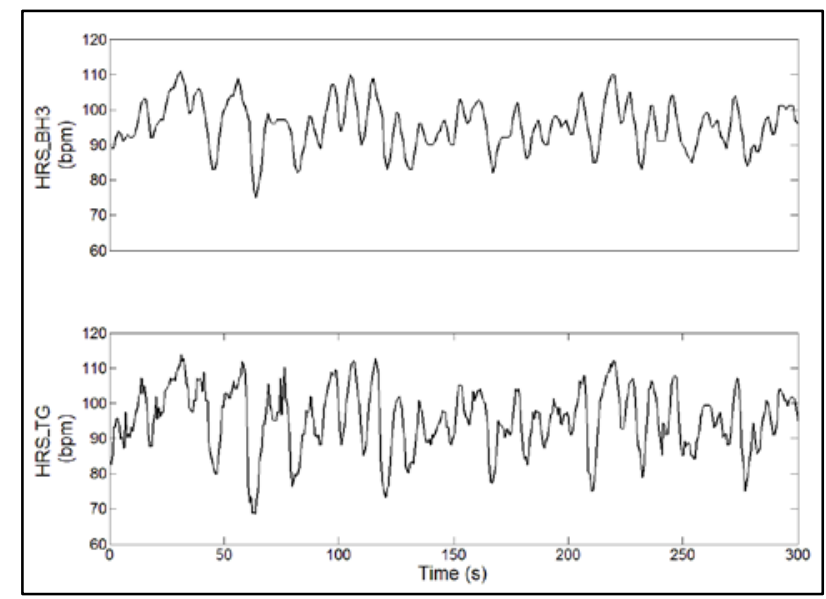

Fig.1. HRS_BH3 (upper panel) vs HRS_TG (lower panel) signals relative to the subject 2 .

being the one to be evaluated. Compared to HRS_TG, HRS_BH3 shows much lower amplitude deviations. A possible cause of this discrepancy could be the $1 \mathrm{~Hz}$ resampling procedure necessarily performed to get HRS_BH3 since HRS_TG is by definition sampled at a frequency that corresponds to the subject's MHR (on average $73 \mathrm{bpm}$ and thus $1.2 \mathrm{~Hz}$ ). Another possible cause of discrepancy between HRS_BH3 and HRS_TG could

Table 1. HR values (mean \pm standard deviation; bpm) provided by HRS_BH3 and HRS_TG, and differences between HRSD values (HRSD error; bpm) provided by HRS_BH3 and HRS_TG, respectively.

\begin{tabular}{|c|c|c|c|}
\hline Subj & $\begin{array}{c}\text { HRS_BH3 } \\
\text { MHR } \\
\text { (bpm) }\end{array}$ & $\begin{array}{c}\text { HRS_TG } \\
\text { MHR } \\
\text { (bpm) }\end{array}$ & $\begin{array}{l}\text { HRSD error } \\
\text { (bpm) }\end{array}$ \\
\hline 1 & $73.2 \pm 3.8$ & $72.7 \pm 5.5$ & 1.7 \\
\hline 2 & $95.6 \pm 6.8$ & $95.5 \pm 9.0$ & 2.2 \\
\hline 3 & $85.3 \pm 7.5$ & $84.3 \pm 10.5$ & 3.0 \\
\hline 4 & $68.8 \pm 1.5$ & $68.8 \pm 1.9$ & 0.4 \\
\hline 5 & $51.7 \pm 2.6$ & $51.7 \pm 3.7$ & 1.1 \\
\hline 6 & $92.9 \pm 2.7$ & $93.0 \pm 2.9$ & 0.2 \\
\hline 7 & $53.2 \pm 7.7$ & $52.7 \pm 8.5$ & 0.8 \\
\hline 8 & $67.9 \pm 2.5$ & $68.0 \pm 2.7$ & 0.2 \\
\hline 9 & $81.4 \pm 4.1$ & $81.7 \pm 4.5$ & 0.4 \\
\hline 10 & $60.7 \pm 5.9$ & $60.9 \pm 7.1$ & 1.2 \\
\hline
\end{tabular}

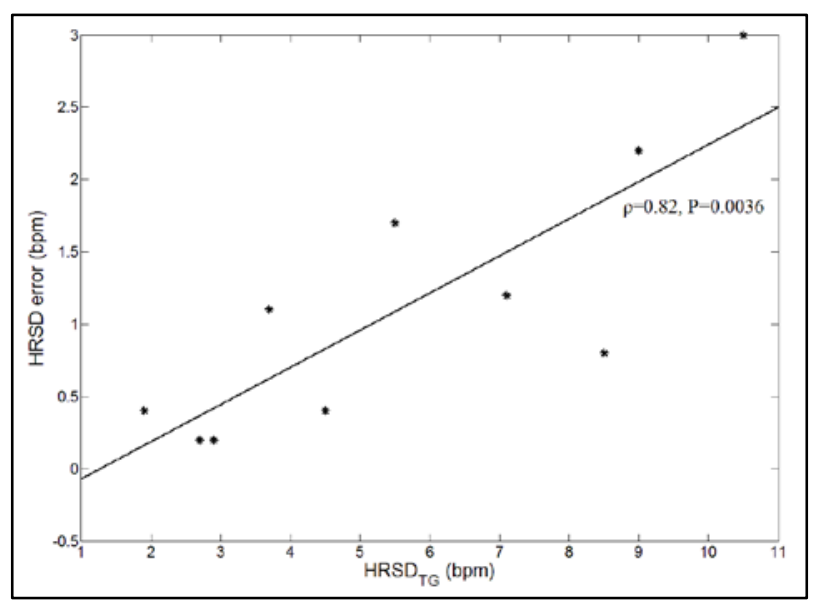

Fig.2. HRSD error as functions of HRSD values provided by HRS_TG with relative regression lines. rifare

be related to errors occurring during R-peak detection. While the correctness of R-peak positions (detected from ECG_BH3) was visually checked, the same cannot be done with the R peaks detected by $\mathrm{BH} 3$ because such data is not available. If there are differences in R-peak detection, these differences will be reflected in HR calculation.

According to our results, HRS_BH3 provides reliable MHR estimations (i.e. comparable to those calculated from HRS_TG) but not HRV values (here measured as HRSD) which is significantly underestimated. Moreover, the underestimation level is strongly correlated to the amount of the variability. Therefore, to light of results, the use of HRS_BH3 is appropriate for sport applications relying on MHR estimations, but not to clinical evaluations based on HRV measurements. Instead, ECG_BH3, although sampled at a frequency $(200 \mathrm{~Hz})$ that is lower than that characterizing modern ECG machine (typically over $1000 \mathrm{~Hz}$ ), can provide useful clinical information, allowing computation of both HR and repolarization parameters such as QT interval [20] Twave alternans [21,22] and f99 [23]. Still, there are several important differences between $\mathrm{BH} 3$ and traditional clinical ECG devices. First of all, the latter provides only one lead ECG whereas the former provide at least 12-lead ECG, and repolarization indexes are known to be lead- dependent [24,25]. In addition, for the way it has been acquired, ECG_BH3 is likely a very noisy recording, so that efficient filtering procedures $[26,27]$ should be applied before computing repolarization parameters [28]. Despite these limitations, ECG_BH3 still provide precious information that could join (not replace) those from traditional electrocardiography in order to improve identification of subjects at increased risk of SCD in the general population. 


\section{Conclusions}

BH3 is a wearable sensor that provides ECG_BH3 and HRS_BH3. If ECG_BH3 may found some complementary clinical utility when fighting SCD in the general population, HRS_BH3 is indicated only for sport applications based on MHR estimations, since HRV measurements were not found to be reliable.

\section{References}

[1] Engelstein ED, Zipes DP. Sudden cardiac death. In: Alexander RW, Schlant RC, Fuster V. The Heart, Arteries and Veins. New York: McGraw-Hill, 1998:1081-112.

[2] Myerburg RJ, Castellanos A. Cardiac arrest and sudden death. In: Braunwald E. Heart Disease, A Textbook of Cardiovascular Medicine. Philadelphia: WB Saunders, 1997:742-79.

[3] Chugh SS, Reinier K, Teodorescu C, et al. Epidemiology of sudden cardiac death: clinical and research implications. Prog Cardiovasc Dis 2008; 51:213-28.

[4] Samol A, Gönes M, Zumhagen S et al. Improved Clinical Risk Stratification in Patients with Long QT Syndrome? Novel Insights from Multi-Channel ECGs. PLoS One 2016; 11(7):e0158085.

[5] Man S, De Winter PV, Maan AC et al. Predictive power of T-wave alternans and of ventricular gradient hysteresis for the occurrence of ventricular arrhythmias in primary prevention cardioverter-defibrillator patients. J Electrocardiol 2011; 44:453-9.

[6] Burattini L, Bini S, Burattini R. Repolarization alternans heterogeneity in healthy subjects and acute myocardial infarction patients. Med Eng Phys 2012; 34:305-12.

[7] Haïssaguerre M, Derval N, Sacher F et al. Sudden cardiac arrest associated with early repolarization. N Engl J Med 2008; 358:2016-23.

[8] Fu GS, Meissner A, Simon R. Repolarization dispersion and sudden cardiac death in patients with impaired left ventricular function. Eur Heart J 1997; 18:281-9.

[9] Jeyaraj D, Haldar SM, Wan X et al. Circadian rhythms govern cardiac repolarization and arrhythmogenesis. Nature 2012; 483:96-9.

[10] Task force of the european society of cardiology and the north american society of pacing and electrophysiology. Heart rate variability guidelines, standards of measurement, physiological interpretation, and clinical use. Eur Heart J Suppl 1996; 17:354-81.

[11] Priori SG, Aliot E, Blomstrom-Lundqvist C et al. Task Force on sudden cardiac death of the European Society of Cardiology. Eur Heart J Suppl 2001; 22:1374-450.

[12] Narayanan K, Chugh SS. The 12-lead electrocardiogram and risk of sudden death: current utility and future prospects. Europace 2015;17 (2):ii7-13.

[13] Refaat MM, Hotait M, Tseng ZH. Utility of the exercise electrocardiogram testing in sudden cardiac death risk stratification. Ann Noninvasive Electrocardiol 2014; 19:311-8.

[14] Katritsis DG, Siontis GC, Camm AJ. Prognostic significance of ambulatory ECG monitoring for ventricular arrhythmias. Prog Cardiovasc Dis 2013; 56(2):133-42.
[15] Marijon E, Tafflet M, Celermajer DS et al. Sports-Related Sudden Death in the General Population, Circulation 2011; 124:672-81.

[16] Borjesson M, Pelliccia A. Incidence and aetiology of sudden cardiac death in young athletes: an international perspective. Br J Sports Med 2009; 43:644-8.

[17] Corrado D, Basso C, Pavei A, et al. Trends in sudden cardiovascular death in young competitive athletes after implementation of a preparticipation screening program. JAMA 2006; 296:1593-601.

[18] Link MS, Estes III M. Sudden cardiac death in the athlete, bridging the gaps between evidence, policy, and practice. Circulation 2012; 125:2511-16.

[19] Pan J, Tompkins WJ. A real-Time QRS detection algorithm. IEEE Transaction on biomedical engineering 1985; 32:230-6.

[20] Azie NE, Adams G, Darpo B et al. Comparing methods of measurement for detecting drug-induced changes in the QT interval: Implications for thoroughly conducted ECG studies. Ann Noninvasive Electrocardiol 2004; 9:166-74.

[21] Burattini L, Bini S, Burattini R. Automatic microvolt Twave alternans identification in relation to ECG interferences surviving preprocessing. Med Eng Phys 2011; 33:17-30.

[22] Burattini L, Bini S, Burattini R. Correlation method versus enhanced modified moving average method for automatic detection of T-wave alternans. Comput Meth Prog Bio 2010; 98:94-102.

[23] C Giuliani, CA Swenne, S Man et al. Predictive Power of f99 Repolarization Index for the Occurrence of Ventricular Arrhythmias. Ann Noninvasive Electrocardiol 2016; 21(2):152-160.

[24] Antlzevitch C. Role of spatial dispersion of repolarization in inherited and acquired sudden cardiac death syndromes. Am J Physiol Heart Circ Physiol 2007; 293:2024-38.

[25] Burattini L, Man S, Burattini R et al. Comparison of standard versus orthogonal ECG leads for T-wave alternans identification. Ann Noninvasive Electrocardiol 2012; 17:130-40.

[26] Agostinelli A, Giuliani C, Burattini L. Extracting a clean ECG from a noisy recording: a new method based on segmented-beat modulation. Computing in Cardiology 2014; 41:49-52.

[27] Agostinelli A, Sbrollini A, Giuliani C et al. Segmented beat modulation method for electrocardiogram estimation from noisy recordings. Med EngPhys 2016; 38(6): 560-8.

[28] Burattini L, Zareba W, Burattini R. Identification of gender-related normality regions for T-wave alternans. Ann Noninvasive Electrocardiol 2010; 15:328-36.

Address for correspondence.

Laura Burattini

Department of Information Engineering

Università Politecnica delle Marche

Via Brecce Bianche, 60131 Ancona, Italy

1.burattini@univpm.it 